\title{
Choice is not the issue. The misrepresentation of healthcare in bioethical discourse
}

\author{
Kari Milch Agledahl, ${ }^{1}$ Reidun Førde, ${ }^{3}$ Åge Wifstad ${ }^{2}$
}

${ }^{1}$ Finnmark Hospital Trust, Norway

${ }^{2}$ Institute of Community Medicine, Faculty of Health Sciences, University of Tromsø, Norway

${ }^{3}$ Section for Medical Ethics, University of Oslo, Norway

\section{Correspondence to}

Kari Milch Agledahl, Finnmark Hospital Trust, Sykehusveien 35 , 9613 Hammerfest, Norway; kari.agledahl@helse-finnmark. no

Received 21 July 2010 Revised 8 October 2010 Accepted 31 October 2010

Published Online First

3 December 2010

\section{ABSTRACT}

The principle of respect for autonomy has shaped much of the bioethics' discourse over the last 50 years, and is now most commonly used in the meaning of respecting autonomous choice. This is probably related to the influential concept of informed consent, which originated in research ethics and was soon also applied to the field of clinical medicine. But while available choices in medical research are well defined, this is rarely the case in healthcare. Consideration of ordinary medical practice reveals that the focus on patient choice does not properly grasp the moral aspects involved in healthcare. Medical decisions are often portrayed as if doctors and patients in confidence confront specific decisions about examinations or treatment, yet the reality often involves many different participants, with decisions being made over time and space. Indeed, most of the decisions are never even presented to patients, as it would be unethical to suggest something that is not medically justifiable. The options patients do confront are somewhat arbitrarily constructed within the narrow framework of both what is deemed to be medically appropriate and how the healthcare system is organised practically. While the autonomy discourse has proven valuable, a failure to distinguish between the fields of medical research and clinical medicine has generated a focus on patient choice that does not reflect what is really at stake in healthcare settings. This is alarming, because the current discourse misrepresents medical practice in a way that actually contributes to bioethical self-delusion.

\section{INTRODUCTION}

The principle of respect for autonomy entered the realm of medicine primarily as a barrier to abuse in the name of science. The disclosure of human exploitation in medical experiments during and after World War II led to calls for the greater protection of research participants, and the Nuremberg Code of 1947 declared that the voluntary consent of the human subject is absolutely essential. ${ }^{1}$ The autonomy principle was carried over to clinical medicine, probably facilitated by human rights movements and a rising discontent with authoritarian and paternalistic doctors. ${ }^{2}$ While autonomy is a multifaceted term in philosophy, signifying authenticity and self-legislative authority, ${ }^{3} 4$ the notion still relates to informed consent in healthcare. Discussions have centred on decision making and patient choice, ${ }^{5-7}$ with movements like 'shared decision making' and 'patient centred medicine'. ${ }^{9}$ In perhaps the most influential book in bioethics, Principles of Biomedical
Ethics, the principle of autonomy is explicitly formulated as respecting autonomous choice, which is now the most common denotation of the concept in bioethics. ${ }^{10}$

Despite its influence, the status of the principle is controversial. The individualistic interpretation of autonomy is said to reflect the specific American context, ${ }^{11}$ while some have pointed out that relational dimensions also have moral significance. ${ }^{12}$ Others assert that the significant emphasis on patient autonomy has led to the underdevelopment of other important moral values, such as beneficence and justice. ${ }^{13}$ Moreover, autonomy is claimed to represent an ideal that fits poorly with clinical practice, since patients are, by definition, unwell and in need of help, and therefore often lack the competence and independence that the principle presupposes. $^{1415}$

While this critique seems appropriate, a central problem is rarely mentioned: while choices in medical research are well defined, this is often not the case in healthcare practice. The Norwegian healthcare system, which serves as the basis for our reflections, is predominantly public, and patients in other countries may have greater influence. Nevertheless, patients cannot generally choose any treatment they desire, and only have a formal right to refuse treatment. These are the conditions of the healthcare system in our society, and they are often underplayed in the autonomy debate. The right to choose presupposes a kind of open choice that is, in fact, rare in healthcare, and this makes 'patient choice' a misleading way of characterising clinical practice.

In this article we reflect upon real-life scenarios taken from every day medical practice. The examples were obtained from a recent study of doctors' medical and moral practice, in which we interviewed doctors from different specialties and observed their daily work. ${ }^{16}$ While the examples are trivial, considering them in light of the choice paradigm reveals an essential shortcoming of the autonomy debate. We aim to demonstrate that choice does not constitute a central moral issue in healthcare, and that the bioethical focus on autonomous choice thus misrepresents the moral aspects at play in this setting.

\section{CHOICE IN CLINICAL PRACTICE}

When considering the issue of respecting patients' autonomous choice we are encouraged to think of cases in which there are alternative procedures available, whereby patients can consider the likely risks and benefits of the different options. Yet such choices are uncommon in practice, and recognising the decisions that patients make autonomously, or unlocked scheme, see http:// jme.bmj.com/site/about/ unlocked.xhtml 
even identifying the options they confront, is actually quite difficult.

Consider the following example from general practice:

The patient, a middle-aged woman, enters the doctor's surgery and greets the doctor, who is a locum for her usual family doctor.

Patient: 'I received a letter that stated that my long-term blood sugar was elevated.'

Doctor: 'HbA1c? Well, then, we should measure your blood pressure and take a glucose tolerance test. There's a danger of developing diabetes.'

The doctor starts to fill in the form for a laboratory requisition.

The patient addresses the doctor with a request; she wants to have her blood sugar followed up. The doctor responds by conducting an examination and explaining the consequences for the patient. Now, you could present this case as an example of how the doctor is respecting the patient's autonomous decision to have her blood sugar investigated. But is this accurate?

Is it reasonable to assert that the patient wishes to have her long-term blood sugar followed up when she does not, in fact, know what it is, what it can lead to, and does not have any symptoms? Who, in this particular case, wishes a change in her blood sugar? Her family doctor who first prescribed the test, the nurse who actually took it, the lab personnel who analysed the sample or the locum doctor she now encounters? In this example it is not a single individual or decision that brought this woman to the doctor, but a series of decisions made by several people over a longer period of time. Some could argue that arranging the initial contact with her family doctor is an autonomous act, even if her original issue had nothing to do with elevated blood sugar, yet evidence of autonomous choices within the healthcare system is hard to distinguish. The elevated long-term blood sugar is followed up because the medical personnel know that the patient has an increased risk of developing diabetes. The patient trusts the doctors and cooperates to the necessary tests. If you try to describe this medical practice in terms of autonomous choice, the result is a rather creative report.

Some of the difficulties with the concept of autonomous choice in clinical practice relate to the fact that what we normally perceive as a single action actually involves many different participants. A medical examination or treatment involves the patient and the doctor and other professionals, such as radiologists, nurses or lab personnel, who each have a limited role to play in the procedure. Some of these sub-actions may perhaps be described as merely procedural, but as individuals with their own will and responsibility, these people add to the complexity of the notion of autonomous action. Even to speak of 'the doctor' as one person can be misleading, since doctors vary in their liability to a particular patient. The doctor who orders a test may not be the one who gets the result. The doctor who admits the patient to hospital may not be the one doing ward rounds the next day, and who continues the treatment already ordered. Doctors confer with other doctors, including their superiors, specialists and colleagues, in such a way that the decisions that an individual doctor makes are often the result of balancing a number of opinions. Moreover, a series of 'invisible' participants, like instructions, procedures and recommendations direct how the doctor can and will make a decision. Thus, talking about choice in clinical practice rarely reflects a single, detached decision, but instead relates to a series of actions that collectively make up an examination or a treatment. Patient information is derived from examining or talking to patients and also obtained from various clinical personnel, as well as sources like patient records and laboratory tests. Indeed, many decisions are made without the patients even being present, meaning that many aspects of choice are beyond their reach.

The interesting point is not that any action can, in theory, be broken down into lesser sub-actions, but that actions in clinical medicine are essentially divided because they are performed by different people, often at different places and at different points in time. This makes it harder to define who has made the decision, identify who has the moral responsibility and understand what respecting a patient's right to autonomous choice could mean. Clinical practice is better described as a process over time and space in which several participants guide the actions that are taken. Patients are not without influence in this process, since good clinical practice is adjusted to their needs, but opportunities for autonomous choice are, in fact, quite limited.

\section{THE CONSTRUCTION OF CHOICE}

Even though the dynamics of every day clinical work are best understood as a process, patients apparently do still face some concrete decisions about their treatment.

Doctor: 'We have detected that you have a hiatus hernia, which can be the cause of your discomfort. This is something we are able to operate on. When the hernia is open, like now, gastric juice flows up from the stomach into the oesophagus and causes inflammation. We can give you medication to make the gastric content less acidic, but it doesn't prevent food from flowing up the oesophagus. Have you experienced any trouble with gastric juice flooding back as you lay down?'

Patient: 'Yes.'

Doctor: 'You won't get rid of that problem only with medication; it would have to be operated on. But you have to decide what you want to do. This is something you have to judge yourself.'

In this example it is up to the patient to decide whether he wants medication or surgery, and he apparently has an opportunity to make an autonomous decision about his treatment. However, in order to present this as a choice for the patient, the doctor has already made several significant decisions. For instance, he has decided to examine the patient with a gastroscope and ascribe significance to the detection of a hiatus hernia, even though this is a common and often asymptomatic condition. He has judged that the condition can be improved by surgery, but also that surgical intervention is not essential for the patient's medical wellbeing. So, when the choice is finally presented to the patient, a series of significant medical decisions have already been made, and the options are constructed around two alternatives, both of which the doctor believes are medically justifiable. The patient does have a choice, but the options are defined within a narrow framework set by the doctor in advance. Surgery and medication are both evaluated as adequate treatment for the patient's condition, and this is precisely why he is allowed to choose. While this particular choice might be an important one, not every choice is central to patient self-determination.

Doctor: 'It all depends on how we present it to the patient. When we're going to anaesthetise a child, I normally ask: "Do you want the needle in this arm or that?"'

The doctor shows his right and left hand.

Doctor: "Then they declare: "Why, I want it in this hand!" "Certainly," I will say, "You shall have it your way." After all, they aren't allowed to decide whether or not they are injected.' 
This patient's choice is independent and autonomous. Still, since the doctor has already made the medical decisions, including the need for a venous catheter, it is obviously irrelevant from a medical point of view and the moral significance thereof is dubious. While this latter case is an example of paternalistic behaviour, possibly justified in this particular setting, the issue is not that some doctors attempt to steer patients' choice, but that the presence of a choice does not in itself ensure empowerment of patients. Explicit choices, like the one between medication and operation, are often somewhat arbitrary since the course of treatment is a process involving many small evaluations and decisions over a period of time.

\section{MEDICAL RELIABILITY}

In forming examination plans and devising treatment regimes, doctors are largely defining their patients' options without their involvement. Conversely, doctors are not simply free to conduct a medical assessment. Clinical medicine is not neutral, but is aimed at helping patients. Doctors cannot just offer any form of treatment; the underlying premise is that the procedure will improve the patient's health. The definition of 'medically appropriate' is founded in an assessment of the health-related benefits for the patient. This definition guides what doctors perceive to be medical decisions, in which patients' opinions are not necessarily parts of the equation. ${ }^{16}$ Medical interventions are intended to improve a patient's health and this directs the clinical process unless the patient actively opposes it.

An internist does his ward rounds and sits down next to a middleaged man who is lying in bed.

Doctor: 'The CT-scan you had looks fine. We haven't discovered any cancer. The only thing we could find was a small lymph node in your stomach, about $1.5 \mathrm{~cm}$. They can normally be up to $1.5 \mathrm{~cm}$, and so it's borderline.'

Patient: 'What should we do about it then?'

Doctor: 'We'll just follow it closely, to make sure that it doesn't grow.'

\section{Patient: 'Couldn't you just remove it surgically?'}

Doctor: 'No! It's far too small; the surgeons wouldn't be able to find it. In any event, we don't really know that it's anything significant. We won't expose you to such a major operation when it may not be necessary.'

Naturally, the patient is concerned about the enlarged lymph node and wants to have it removed. But the doctor does not present this option, because he does not consider it medically appropriate to expose the patient to the surgical risk. The medical considerations are an analysis of what is best for the patient's overall health, and this assessment is not affected by his fear of cancer.

If it were clear and unambiguous which treatment will produce the best outcome for the patient, it would not be medically justifiable to offer anything else. Doctors' opportunities to present options are restricted by the confines of medical reliability, meaning that only decisions with a limited or uncertain effect are on offer.

\section{HEALTHCARE STRUCTURE}

In addition to the prerequisites of medical justifiability, doctors have to ensure that the decisions are made in line with the particular organisation of the healthcare system.
The patient, a young man, telephones the surgeon on call at the local hospital, complaining of pain after a surgical procedure. The doctor proposes that he comes in for an examination, but the patient wants to be seen at the district general hospital, insisting that he is in severe pain.

Doctor: 'We can't refer you just because you tell us that you are in a lot of pain, we have to see whether the wound is about to close or has got worse... When we've examined you, we may refer you or call the district general hospital to ask them their opinion.'

Patient: 'But couldn't I speak to those surgeons directly?'

Doctor: 'Well... yes, you could certainly call the hospital and ask for them yourself, but I'm not sure that you will be allowed to speak to them. Or else your family doctor could refer you straight to the district general hospital, but in that scenario there would be a considerable waiting list to get an appointment. It will be quicker if we could examine you first and refer you based on our examination.'

The patient is determined to be referred to the specialists at the district general hospital. When his demands are not met, it is not primarily because of a paternalistic doctor, but due to the organisation of this healthcare system. To refer a case to a specialist, the doctor must provide a medical rationale for the referral that is based on an examination of the patient, and this limits what can be done. Attempts to bend the rules might have inadvertent consequences, and a direct referral to the hospital of choice runs the risk of being refused.

The specific rules and organisational structures differ between countries, and especially between a commercially financed healthcare and a public one. Still, healthcare as an organisational system is rather strictly structured and shares many common features in all Western countries. Specialist, modern medicine is not only conducted by doctors, but also by nurses, radiologists, secretaries and directors, each with separate fields of work that interact in a closely regulated manner, including procedures of referral to different specialities and systems for controlling medical expenses. Patients' requests have to be dealt with within the frameworks of this form of organisation. The way of dividing medical specialties and departments based on bodily organs like the lungs, heart, skeletal and immune systems-as well as the division between psychiatric and somatic diseasesmirror the structure of current scientific, medical knowledge that, in turn, controls how patients' health complaints are addressed. The healthcare system also involves more than simply dealing with medical questions; it is a social institution in which administrative and juridical regulations guide medical processes to a large degree. A patient's record is an important tool for clinicians, but it also has administrative and juridical purposes. Practical circumstances govern many medical decisions. What equipment and personnel are available? What will the proposed tests cost? These are concrete and practical issues that play a central role in the assessment of patients. More often than we would normally think of, medical decisions are directed by practical arrangements, and the organisation of healthcare has profound impact on the options and choices open to both doctors and patients.

\section{DISCUSSION}

Our description of clinical practice is not controversial, and will be familiar to anyone who has ever worked in healthcare. Yet this practical knowledge seems to be ignored as we move into the field of bioethical discourse, and what 'everybody really 
knows' is often not properly considered. Patients' right to autonomous choice is upheld as an ideal although the options of both the patients and the doctors are very limited. The autonomy discourse is clouded by the fact that many do not clearly differentiate between clinical medicine and medical research. While of major importance when it comes to securing voluntary participation in medical research, the principle of autonomous choice is transferred to another branch of medicine that has a different objective. Having independent, competent participants and the time to deliberate, which form the template of informed decision-making in medical research, is far less prevalent in clinical medicine. Here, patients are sick, dependent on assistance and choices have to be made even if the time for reflection is limited. In the healthcare setting, choices are often neither explicit nor available.

On the basis of empirical studies, researchers have pointed to the gap between the bioethical discourse of 'end-of-life-decisions' and the reality in which these patients find themselves. ${ }^{17}$ The problem becomes especially evident concerning the terminally ill, who often are portrayed as facing life and death choices, while the decisions are, in fact, almost meaningless, medically speaking. Several authors have criticised what they call 'the choice paradigm' and 'the illusion of choice'. ${ }^{18} 19$ The consequences of individual decisions have often proved to be difficult for patients to comprehend, which some have taken to indicate that patients do not want to make autonomous choices. ${ }^{20}$ Perhaps it instead reflects the fact that particular decisions cannot be considered in isolation from the rest of the patient's treatment, and that healthcare must be understood as a continuous and partially routine form of praxis. ${ }^{21}$ Autonomous choices play a marginal part in clinical practice.

Nevertheless, there is no doubt that the focus on patient autonomy has affected the development of clinical medicine. Even if choices in healthcare are partly constructed and coincidental, the right to refuse treatment is fundamental and important, and this insight is gained mostly from research ethics. Perhaps the main contribution of the discourse on autonomous choice is the focus on the central position of patients in healthcare. Yet it is not easy to capture the moral sense of respecting autonomous choice in clinical practice without relating it to respect for the persons making these choices. Indeed, our analysis does not dismiss the relevance of autonomy in clinical care, but questions the current overriding focus on choice. ${ }^{22}$ Respecting patients as autonomous persons is far more complex than eliciting choices and acknowledging informed consent. In the prior case with the anaesthesiologist, the moral significance of the patient's autonomous choice is disputable, but the doctor's effort to build a relationship with the patient could still be seen as an attempt to respect the child as a person. Respecting patients as autonomous persons is an important moral issue in clinical work, but it is misleading to relate this respect to the degree of choosing.

The prevailing focus on patient choice has created a distinct discourse in bioethics that does not reflect what is at stake in medical care. This may distract our attention away from conditions of greater moral significance in clinical practice. More importantly, there is also a danger that the current discourse is creating a gap between medical practice and medical ethics, and that doctors are adapting a language from bioethics that does not properly portray their sense of moral responsibility. Some empirical studies already suggest that doctors explicitly support patients' right to autonomous choice, but actually pay little attention to their opinions in clinical practice. ${ }^{23-25}$ If doctors' moral concepts do not correlate with their moral actions, bioethics' directives will have no impact. Worse still, it makes clinical work all the more impenetrable for bioethicists, outsiders, and even for the doctors involved. The emphasis on autonomous choice may create a self-delusion where the upheld ideal is not possible, or even desirable, to achieve in practice. Instead of providing moral guidance, such discussions actually alienate doctors' moral reflections and contribute to workrelated frustration. Philosophical reflections on healthcare make an essential contribution to a moral medical practice, but require that these reflections take account of the premises of clinical work. As clinical medicine is a complex discipline loaded with meaning, empirical studies are crucial for bioethics, and systematic observation might reveal unexpected elements that would otherwise be lost in description. How medical work is portrayed shapes how we think about, understand and judge the moral aspects involved in caring for patients.

Funding The Northern Norway Regional Health Authority, Helse Nord RHF, 8038 Bodø, Norway. Finnmark Hospital Trust, Helse Finnmark HF, Sykehusveien 35, 9615 Hammerfest, Norway.

\section{Competing interests None.}

Provenance and peer review Not commissioned; externally peer reviewed.

\section{REFERENCES}

1. Weindling $\mathbf{P}$. The origins of informed consent: the International Scientific Commission on medical war crimes, and the Nuremberg code. Bull Hist Med 2001;75:37-71.

2. Jonsen AR. The Birth of Bioethics. New York: Oxford University Press, 1988:332-8.

3. O'Neill 0. Autonomy and Trust in Bioethics. Cambridge: Cambridge University Press, 2002:83-6.

4. van Willigenburg $\mathbf{T}$, Delaere P. Protecting autonomy as authenticity using Ulysses contracts. J Med Philos 2005;30:395-409.

5. Veatch RM. Implied, presumed and waived consent: the relative moral wrongs of under- and over-informing. Am J Bioeth 2007;7:39-41.

6. Sreenivasan G. Does informed consent to research require comprehension? Lancet 2003;362:2016-18

7. Maclean AR. Advance directives, future selves and decision-making. Med Law Rev 2006;14:291-320.

8. Emanuel EJ, Emanuel LL. Four models of the physician-patient relationship. JAMA 1992;267:2221-6.

9. Sandman L, Munthe $\mathrm{C}$. Shared decision making, paternalism and patient choice. Health Care Anal 2010;18:60-84.

10. Beauchamp TL, Childress JF. Principles of Biomedical Ethics. 6th edn. New York: Oxford University Press, 2009:13,100.

11. Holm S. Not just autonomy - the principles of american biomedical ethics. J Med Ethics 1995;21:332-8.

12. Callahan D. Individual good and common good: a communitarian approach to bioethics. Perspect Biol Med 2003:46:496-507.

13. Veatch RM. Autonomy's temporary triumph. Hastings Cent Rep 1984;14:38-40.

14. Ackerman T. Why doctors should intervene. Hastings Cent Rep 1982;4:14-17.

15. Pellegrino ED. Toward a reconstruction of medical morality. Am J Bioeth 2006;6:65-71. (Originally published in J Med Humanit Bioeth, 1987;8:7-18.)

16. Agledahl KM, Førde R, Wifstad $\AA$. Clinical essentialising: a qualitative study of doctors' medical and moral practice. Med Health Care and Philos 2010;13:107-13.

17. Drought TS, Koenig BA. 'Choice' in end-of-life decision making: researching fact or fiction? Gerontologist 2002:42(Spec no 3):114-28.

18. Slomka J. The negotiation of death: clinical decision making at the end of life. Soc Sci Med 1992;35:251-9.

19. Sullivan MD. The illusion of patient choice in end-of life-decisions. Am J Geriatr Psychiatry 2002;10:356-72.

20. Schneider CE. The practice of autonomy and the practice of bioethics. J Clin Ethics 2002:13:72-7.

21. Kukla R. Conscientious autonomy: displacing decisions in healthcare. Hastings Cent Rep 2005:35:34-44.

22. Joffe S, Manocchia M, Weeks JC, et al. What do patients value in their hospital care? An empirical perspective on autonomy centred bioethics. J Med Ethics 2003:29:103-8.

23. Lawrence RE, Curlin FA. Autonomy, religion and clinical decisions: findings from a national physician survey. J Med Ethics 2009;35:214-18.

24. Karnieli-Miller 0, Eisikovits Z. Physician as partner or salesman? Shared decision-making in real-time encounters. Soc Sci Med 2009;69:1-8.

25. Deep KS, Griffith CH, Wilson JF. Communication and about life-sustaining treatment: examining the experiences of resident physicians and seriously-ill hospitalized patients. J Gen Intern Med 2008;23:1877-82. 